\title{
Correlation of matrix metalloproteinase suppressor genes RECK, VEGF, and CD105 with angiogenesis and biological behavior in esophageal squamous cell carcinoma
}

\author{
Sheng-Lei Li, Dong-Ling Gao, Zhi-Hua Zhao, Zong-Wen Liu, Qiu-Min Zhao, Jin-Xia Yu, Kui-Sheng Chen, \\ Yun-Han Zhang
}

\begin{abstract}
Sheng-Lei Li, Zhi-Hua Zhao, Dong-Ling Gao, Zong-Wen Liu, Qiu-Min Zhao, Jin-Xia Yu, Kui-Sheng Chen, Yun-Han Zhang, Department of Pathology, The First Affiliated Hospital Zhengzhou University; He'nan Key Laboratory of Tumor Pathology, Zhengzhou 450052, He'nan Province, China

Supported by The "Tenth Five-Year Plan" Research Foundation for the Key Constructional Project ("211 Project") of Zhengzhou University, He'nan Province, China

Correspondence to: Yun-Han Zhang, Department of Pathology, The First Affiliated Hospital, Zhengzhou University; Henan Key Laboratory of Tumor Pathology, Zhengzhou 450052, He'nan Province, China. yhzhang@zzu.edu.cn

Telephone: +86-371-65168738 Fax: +86-371-66658175

Received: June 4, $2007 \quad$ Revised: September 3, 2007
\end{abstract}

\begin{abstract}
AIM: To explore the expression of reversion inducing cysteine-rich protein with Kazal motifs (RECK), vascular endothelial growth factor (VEGF) and endoglin (CD105) protein and its correlation with occurrence, development, invasion and metastasis in esophageal squamous cell carcinoma (ESCC).
\end{abstract}

METHODS: Streptavidin-peroxidase (SP) immunohistochemistry was used to detect expression of RECK and VEGF in 62 cases of ESCC, 31 cases of adjacent atypical hyperplastic epithelium and 62 cases of normal esophageal epithelium. CD105 Mb was used to assess microvessel density (MVD).

RESULTS: The expression of RECK was closely correlated with histological grade, infiltrative depth and lymphatic metastasis in ESCC $(P<0.05)$. The expression of RECK decreased during cancer development: normal esophageal epithelium $(85.5 \%, 53 / 62)$, adjacent atypical hyperplastic epithelium $(71.0 \%, 22 / 31)$, and carcinoma $(59.7 \%, 37 / 62)$. There was a significant difference among the groups $(P<0.05)$. The expression of VEGF protein was closely correlated with infiltrative depth and lymphatic metastasis in $\operatorname{ESCC}(P<0.05)$. The expression of VEGF protein increased during cancer development: normal esophageal epithelium $(29.0 \%, 18 / 62)$, adjacent atypical hyperplastic epithelium $(54.8 \%, 17 / 31)$, and carcinoma $(67.7 \%, 42 / 62)$. There was a significant difference among the groups $(P<0.05)$. MVDCD105 increased in accordance with histological grade, but there was no significant difference (grade I, $36.92 \pm$ 10.85; grade II, $37.65 \pm 9.50$; and grade III, 38.06 \pm 12.19). The MVDCD105 was closely correlated with infiltration and lymphatic metastasis in $\operatorname{ESCC}(P<0.05)$. The expression of RECK was inversely correlated with the expression of VEGF and CD105.

CONCLUSION: RECK, VEGF and CD105 play important roles in the infiltration, metastasis and carcinogenesis in esophageal carcinoma. Angiogenesis in ESCC may be promoted by over-expression of CD105.

\section{(c) 2007 WJG. All rights reserved.}

Key words: Reversion inducing cysteine rich protein with Kazal motifs; Vascular endothelial growth factor; CD105; Esophageal squamous cell carcinoma; Immunohistochemistry; Microvessel density

Li SL, Gao DL, Zhao ZH, Liu ZW, Zhao QM, Yu JX, Chen $\mathrm{KS}$, Zhang $\mathrm{YH}$. Correlation of matrix metalloproteinase suppressor genes RECK, VEGF and CD105 with angiogenesis and biological behavior in esophageal squamous cell carcinoma. World J Gastroenterol 2007; 13(45): 6076-6081

http://www.wjgnet.com/1007-9327/13/6076.asp

\section{INTRODUCTION}

Reversion inducing cysteine-rich protein with Kazal motifs (RECK) is a recently discovered tumor suppressor gene with a special function of inhibiting matrix metalloproteinase (MMP) expression and activity, which serves as an MMP inhibitor ${ }^{[1]}$. Expression of the RECK gene is closely related to tumor invasion and metastasis and angiogenesis. Previous studies indicate that the level of RECK gene expression is inversely correlated to tumor invasiveness in liver cancer, pancreatic cancer, mammary cancer and pulmonary carcinoma, and for patients with higher RECK gene expression, the prognosis is sometimes apparently better than that of patients with low expression $^{[2-5]}$. No studies have been published in China or abroad on the correlation of the RECK gene with invasion and metastasis of esophageal cancer, and the relationship between RECK, vascular endothelial growth factor (VEGF) 
and endoglin (CD105) expression. The streptavidinperoxidase (SP) immunohistochemistry method was used to perform a combined test on expression of RECK, VEGF and CD105 gene in tissues from 62 cases of esophageal squamous cell carcinoma (ESCC), 31 cases of para-carcinoma atypical hyperplasia, and 62 specimens of normal esophageal mucous membrane, to establish the role of RECK, VEGF and CD105 in the generation and development of esophageal cancer, so as to ascertain the molecular index for early diagnosis and prognosis judgment.

\section{MATERIALS AND METHODS}

\section{Materials}

Resection specimens from 62 cases of esophageal cancer were collected from the Municipal Cancer Hospital of Anyang, Henan Province, China from 26 February to 16 March, 2006, which is one of the most epidemic regions for esophageal cancer. No patients had a history of chemotherapy, radiotherapy or immunotherapy. The specimens were taken from 36 male and 26 female patients aged $38-75$ years (average $60.6 \pm 9.5$ ), who were all verified to have ESCC by histopathological examination. The histological grading included Class I (15 cases), Class II ( 25 cases) and Class III (22 cases); 20 cases were accompanied with lymphatic metastasis, and 42 cases had no lymphatic metastasis. The depth of invasion was divided into two groups that consisted of seven cases with invasion of the superficial muscularis, and 55 with invasion of the deep muscularis or fibrous membrane. All the samples were taken from within $3 \mathrm{~cm}$ of the tumor focus, as well as from three areas of distal normal mucous membrane, and were fixed with $40 \mathrm{~g} / \mathrm{L}$ paraformaldehyde solution, normally dehydrated, embedded in paraffin, and serial sections were cut to a thickness of 4-6 $\mu \mathrm{m}$, and used for hematoxylin and eosin and immunohistochemistry staining. Mouse anti-human RECK monoclonal antibody $(\mathrm{mAb})$ and anti-human VEGF mAb were all purchased from Santa Cruz Biotechnology (Santa Cruz, CA, USA), and mouse anti-human CD105 single clone antibody and the SP immunohistochemistry kit were purchased from Beijing Zhongshan Golden Bridge Biotech Development (China).

\section{Methods}

The SP immunohistochemistry SP method was employed. RECK, VEGF and CD105 mAbs were diluted 1:100, stained with diaminobenzidine, and counterstained with hematoxylin, strictly in accordance with the instructions, and PBS solution was used as a negative control replacing primary antibody. RECK- and VEGF-positive signals all show brown granule-like materials that are located in the cytoplasm. Under a high-power magnifying glass, five fields of vision (FOVs) were randomly selected (for each FOV, there were no fewer than 200 cells), and the results were interpreted in accordance with the percentage of cells and depth of $\operatorname{stain}^{[6]}$. (1) Scored in accordance with depth of staining of cells in a section: 0 , no cell coloration; 1 , light yellow; 2, brown; 3, tan. (2) Scored in accordance with the percentage of positive cells in like-kind cells: $1,<30 \% ; 2$, $30 \%-70 \% ; 3>70 \%$. The product of (1) and (2) was used as the total score, where $0-1$ indicates a negative score $(-)$, $2-3$ a weak positive score $(+)$, and $\geqslant 4$ a positive score $(++)$. Tumor MVD measurement was done using the methods reported by Weidner ${ }^{[7]}$, i.e. any brown endothelial cell or cell cluster is used as a vessel. We first observed all the FOVs for a given section under a low-power magnifying glass to find the highest density area of tumor vessels, and then we counted the number of microvessels in three FOVs under a high-power magnifying glass; the average values were then used as the MVD.

\section{Statistical analysis}

SPSS 10.0 statistical software was used and the $\chi^{2}$ test, single factor analysis of variance, $t$ test were applied, correlation test was applied, Spearman correlation analysis. The test level was $\alpha=0.05$.

\section{RESULTS}

\section{RECK expression in ESCC tissues and correlation with} clinical and biological behavior

RECK expression was mainly located in the cytoplasm of tumor cells, and appeared as light to dark yellow (Figure 1A). RECK expression increased sequentially as ESCC developed: normal tissue $(59.7 \%, 37 / 62)$, paracarcinoma atypical hyperplastic tissue $(71.0 \%, 22 / 31)$, and ESCC (85.5\%, 53/62), and comparison between the groups indicated a significant difference $\left(\chi^{2}=10.331\right.$, $P<0.01$ ) (Table 1). RECK expression was related to histological grading, invasion depth and lymphatic metastasis (the respective values of $\chi^{2}$ were 10.422, 8.550 and 4.751; average $P<0.05$ ) (Table 2).

\section{VEGF expression in ESCC tissues and correlation with clinical and biological behavior}

VEGF staining was located in the cytoplasm, and appeared as light to dark yellow (Figure 1B). VEGF expression decreased sequentially as ESCC developed: normal mucous membrane $(67.7 \%, 42 / 62)$, para-carcinoma atypical hyperplastic tissue $(54.8,17 / 31)$, and carcinoma $(29.0 \% 18 / 62)$; and comparison between the groups indicated a significant difference $\left(\chi^{2}=18.994, P<0.05\right)$ (Table 1). VEGF expression was not related to histological grading $(P>0.05)$, but was related to depth of invasion and lymphatic metastasis (the respective values of $\chi^{2}$ were 10.319 and 6.693; average $P<0.05)$ (Table 2).

\section{Correlation of MVDCD105 with differentiation and metastasis in ESCC}

CD105 expression was mainly located in the cytoplasm of vascular endothelial cells of tumor stroma, and appeared as light to dark yellow granules (Figure 1C). In grade I, II and III ESCC tissues, MVDCD105 tended to increase as the degree of cancer tissue differentiation decreased: grade I , $37.87 \pm 3.60$; grade II , $37.44 \pm 3.99$; and grade III, $39.00 \pm 4.47$ ), but there was no significant difference between the results $(F=0.885, P>0.05)$ (Table 1$)$. In cancer tissues with lymphatic metastasis, MVD (41.00 \pm 

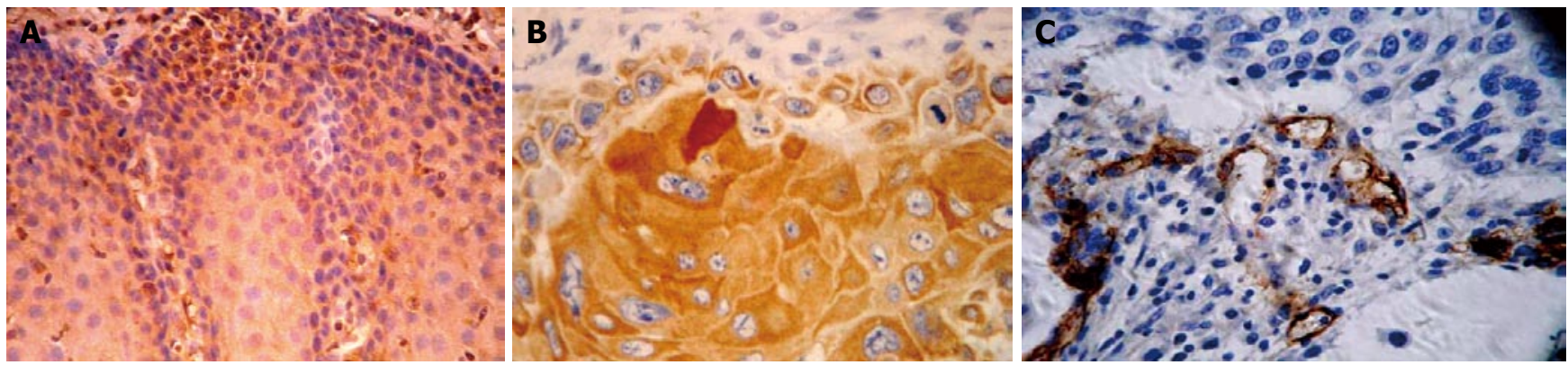

Figure 1 A: Expression of RECK in the normal esophageal epithelium; B: Expression of VEGF in ESCC; C: Expression of CD105 in ESCC (SP, × 400).

Table 1 RECK and VEGF expression in ESCC, atypical hyperplasia and normal mucous membrane tissues

\begin{tabular}{|c|c|c|c|c|c|c|c|c|c|c|c|}
\hline & \multirow[b]{2}{*}{$n$} & \multicolumn{3}{|c|}{ RECK } & \multirow[t]{2}{*}{$\chi^{2}$} & \multirow[t]{2}{*}{$P$} & \multicolumn{3}{|c|}{ VEGF } & \multirow[t]{2}{*}{$\chi^{2}$} & \multirow[t]{2}{*}{$P$} \\
\hline & & - & + & Positive ratio (\%) & & & - & + & Positive ratio (\%) & & \\
\hline Normal mucous membrane tissues & 62 & 9 & 53 & 85.5 & & & 44 & 18 & 29 & & \\
\hline Atypical hyperplasia & 31 & 9 & 22 & 71.0 & 10.331 & 0.006 & 14 & 17 & 54.8 & 18.994 & 0.000 \\
\hline ESCC & 62 & 25 & 37 & 59.7 & & & 20 & 42 & 67.7 & & \\
\hline
\end{tabular}

Table 2 Correlation of RECK and VEGF expression with clinical and biological behavior of ESCC

\begin{tabular}{|c|c|c|c|c|c|c|c|c|c|c|c|}
\hline & \multirow[t]{2}{*}{$n$} & \multicolumn{3}{|c|}{ RECK } & \multirow[t]{2}{*}{$\chi^{2}$} & \multirow[t]{2}{*}{$P$} & \multicolumn{3}{|c|}{ VEGF } & \multirow[t]{2}{*}{$\chi^{2}$} & \multirow[t]{2}{*}{$P$} \\
\hline & & - & + & Positive ratio (\%) & & & - & + & Positive ratio (\%) & & \\
\hline \multicolumn{12}{|l|}{ Histology grading } \\
\hline I & 15 & 1 & 14 & 93.3 & & & 8 & 7 & 46.7 & & \\
\hline II & 25 & 11 & 14 & 56 & 10.422 & 0.005 & 7 & 18 & 72.0 & 4.014 & 0.134 \\
\hline III & 22 & 13 & 9 & 40.9 & & & 5 & 17 & 77.3 & & \\
\hline \multicolumn{12}{|l|}{ Invasion depth } \\
\hline Superficial muscularis & 7 & 0 & 7 & 100 & & & 6 & 1 & 14.3 & & \\
\hline Deep muscularis & 55 & 25 & 30 & 54.5 & 8.550 & 0.021 & 14 & 41 & 74.5 & 10.319 & 0.001 \\
\hline \multicolumn{12}{|l|}{ Lymph metastasis } \\
\hline $\mathrm{N}$ & 42 & 13 & 29 & 69 & 4.751 & 0.029 & 18 & 24 & 57.1 & 6.693 & 0.010 \\
\hline $\mathrm{Y}$ & 20 & 12 & 8 & 40 & & & 2 & 18 & 90.0 & & \\
\hline
\end{tabular}

3.26) was higher than that without metastasis (36.33 \pm 3.76). MVD (38.80 \pm 3.60$)$ of cancer tissues with invasion of the deep muscularis was higher than that of the superficial muscularis $(32.57 \pm 3.46)$, and the difference was significant (Table 3).

\section{Correlation of RECK, VEGF and CD105 expression in ESCC}

RECK expression was inversely correlated to that of VEGF. In tissues with positive RECK expression, VEGF expression rate was $51.4 \%(19 / 37)$, and in tissues negative for RECK expression, VEGF expression rate was $92.0 \%$ $(23 / 25)$. Comparison between the groups indicated a significant difference $\left(\gamma_{\mathrm{s}}=-0.427, P<0.01\right)$. In tissues with positive RECK expression, MVDCD105 was 35.76 \pm 9.42 , and tissues negative for RECK expression, MVDCD105 was $41.59 \pm 10.80$. Comparison between the groups indicated a significant difference $(t=-2.969, P<$ 0.01) (Table 4).

\section{DISCUSSION}

The RECK gene were discovered by Takahashi et al ${ }^{[1]}$ in NIH3T3 cell lines transfected by the $\mathrm{v}$-Ki-Ras gene, which is located on chromosome 9p13-p12 and encodes a membrane-anchored glucose protein with a relative molecular mass of 110000 . RECK gene has high expression in normal tissues, but no expression in various tumor cell lines and cells affected by cancer genes such as Ras. Many cancer genes such as ras, fos and myc can all decrease the expression of the RECK gene ${ }^{[8,9]}$, which indicates that the RECK gene may be a negatively adjusted target jointly acted upon by cancer genes, and the proper expression of the RECK gene can inhibit angiogenesis ${ }^{[10-12]}$. It has been shown that the RECK gene is closely related to the prognosis of liver cancer, pancreatic cancer and mammary cancer, and prognosis of patients positive for RECK expression is better than that of those with negative expression $^{[2-5]}$. The effects of RECK during the course of tumor generation and metastasis may be more widespread than has been discovered to date. It is now considered that RECK may affect tumor invasion and metastasis through inhibiting tumor angiogenesis, and is thus a cancerinhibiting gene ${ }^{[12-14]}$. The results of this experiment indicate that the RECK gene is expressed in normal esophageal tissues, esophageal para-carcinoma atypical hyperplastic 
Table 3 Correlation of CD105 expression with the clinical and biological behavior of ESCC (reciprocal) (mean \pm SD)

\begin{tabular}{lrccc}
\hline & \multicolumn{4}{c}{ CD105 } \\
\cline { 2 - 5 } & $\boldsymbol{n}$ & MVD & $\boldsymbol{t} / \boldsymbol{F}$ & $\boldsymbol{P}$ \\
\hline Histology grading & 15 & $37.87 \pm 3.60$ & & \\
$\quad$ I & 25 & $37.44 \pm 3.99$ & $F=0.885$ & 0.418 \\
II & 22 & $39.00 \pm 4.47$ & & \\
III & & & & \\
Invasion depth & 7 & $32.57 \pm 3.46$ & -4.326 & 0.000 \\
$\quad$ Superficial muscularis & 55 & $38.80 \pm 3.60$ & & \\
$\quad$ Deep muscularis & & & & \\
Lymph metastasis & 42 & $36.33 \pm 3.76$ & 4.760 & 0.000 \\
$\quad$ N & 20 & $41.00 \pm 3.26$ & & \\
$\quad$ Y & &
\end{tabular}

tissues and cancer tissues, but its expression level in cancer tissue is significantly lower. This indicates that tumors with low RECK expression have greater invasive capacity.

VEGF is a polypeptide cell factor discovered in recent years (also called vascular permeability factor), which has a double function: (1) it directly stimulates vascular endothelial cell reproduction through its receptor, and induces production of the proteolytic enzyme interstitial collagenase and tissue factor to promote angiogenesis; and (2) it increases vascular permeability, promotes exosmosis of fibrinogen to cause tumor interstitial edema and extracellular matrix changes, and consequently provides a basis for tumor invasion and metastasis ${ }^{15,16]}$. VEGF is an angiogenic factor that has been studied at the most with the most specific effect at present, and which is related to generation and metastasis of various human tumors ${ }^{[17-23]}$. The results of this study show that VEGF expression is inversely related to ESCC lymphatic metastasis, i.e. the positive protein rate of VEGF for the lymph metastasis group significantly higher than that of no lymph metastasis group, which indicates that the positive protein expression of VEGF maybe correlated with metastasis of ESCC lymph. The results of this study also show that VEGF expression is not obviously related to the degree of tumor differentiation, which indicates that VEGF expression is not related to ESCC histology.

CD105 is a gene located in human chromosome 9q34, which is a homotype dipolymer membrane glycoprotein with a molecular mass of $180 \mathrm{kDa}$, participating in signal transmission inverting growth factor $\beta$ (TGF- $\beta$ ) receptor and angiogenesis. Previous studies indicate that strong expression of CD105 in tumor-related, newly generated vascular endothelium is a more accurate index for judging endothelial reproductive state, and it is closely related to tumor generation and metastasis ${ }^{[24-27]}$. Contrasting with endothelial cell markers such as CD31, CD34 and VIII factor-related antigens, the difference is that CD105 as a marker of angiogenesis is only strongly expressed in vascular endothelial cells of tumor tissues at the reproduction stage (i.e. newly generated vascular endothelium), but it is not expressed in the blood vessels of normal tissues ${ }^{[28-30]}$. A quantitative test has been carried out on the MVD of newly generated vessels in ESCC with anti-CD105 single clone antibody, the results of which indicated that MVD is positively correlated with depth
Table 4 Correlation of RECK, VEGF and CD105 expressions in ESCC (mean \pm SD)

\begin{tabular}{cccrccccc}
\hline \multirow{2}{*}{ RECK } & $\boldsymbol{n}$ & \multicolumn{2}{c}{ VEGF } & $\gamma_{\mathbf{s}}$ & $\boldsymbol{P}$ & MVD & $\boldsymbol{t}$ & \multirow{2}{*}{$\boldsymbol{P}$} \\
\cline { 3 - 8 } & & $\boldsymbol{+}$ & $\boldsymbol{-}$ & & & & & \\
\hline+ & 37 & 19 & 18 & -0.427 & 0.001 & $36.00 \pm 3.80$ & -2.969 & 0.004 \\
- & 25 & 23 & 2 & & & $39.10 \pm 3.86$ & & \\
\hline
\end{tabular}

of invasion and lymphatic metastasis in ESCC. That is, the MVD of tumors with deep invasion is significantly higher than that with superficial invasion, and the MVD of tumors with lymphatic metastasis is significantly higher than that of those without metastasis; however, MVD is not related to histological grading. Thus, the fixed vessel quantity in tumors is considered as a significant and separate prognosis index. The level of angiogenesis in cancer can be evaluated through MVD measurement ${ }^{[31]}$, and MVD measurement in ESCC may help to judge its potential for invasion and metastasis. The MVD in tumor tissues was tested with CD105, the results of which indicate that RECK expression is inversely related to tumor angiogenesis; further, MVDCD105 for tumors with high RECK expression is obviously lower than that for those with low RECK expression, which indicates that RECK can inhibit angiogenesis. There is a co-adjustable mechanism between MVDCD105 and RECK.

Angiogenesis inhibition by RECK has also been verified in some clinical studies that have discovered that MVD in tumor tissues is inversely related to RECK expression ${ }^{[10,32]}$. However, such an inverse correlation only occurs when the expression of VEGF is much higher, i.e. for tumors with higher expression of VEGF, the influence of RECK also increases, which indicates that RECK can inhibit VEGFinduced angiogenesis. The results indicate that RECK expression is closely related to tumor prognosis. Also, the inhibitory effects of RECK on tumor angiogenesis have a certain pertinence. The results of this study indicate that positive expression of RECK is inversely correlated with VEGF expression and MVD. In cases with negative RECK expression, VEGF expression and MVD are significantly higher than in cases with positive RECK expression $(P<0.05)$. This indicates that decreasing or losing RECK expression may increase VEGF expression, which consequently promotes tumor angiogenesis, and provides the conditions for the generation and metastasis of ESCC. This study also discovered that VEGF expression is consistent with MVD, i.e. if VEGF expression is high, MVD will rise accordingly $(P<0.05)$, and the VEGF's positive stained cells at the front of tumor infiltration, which are consistent with CD105's expression positions, which further verifies that VEGF is an angiogenesis factor with specific effects, and can specifically promote tumor angiogenesis. This study shows that decreasing or losing RECK expression and increasing VEGF expression are two of the significant events during the generation and development of ESCC, and RECK, as a cancer-inhibiting gene, may inhibit angiogenesis in ESCC, through affecting the signal transmission path of VEGF expression. The combined test on RECK, VEGF and MVD can be used as an 
objective index to determine the invasion and metastasis capabilities of ESCC, which is of great significance for judging prognosis.

\section{COMMENTS}

\section{Background}

The RECK gene was discovered by Takahashi et al in NIH3T3 cell lines transfected by the V-Ki-Ras gene, which plays an important role in regulating MMPs and participating in tumor invasion, metastasis and angiogenesis.

\section{Research frontiers}

No studies have been published in China or abroad on the correlation of the RECK gene with invasion and metastasis of esophageal cancer, and the relationship between RECK and expression of VEGF and CD105.

\section{Related publications}

Expression of RECK gene is closely related to tumor invasion and metastasis and angiogenesis. Previous studies indicate that RECK gene expression is inversely correlated with tumor invasiveness in liver cancer, pancreatic cancer, mammary cancer and pulmonary carcinoma, and for patients with higher RECK gene expression, the prognosis is apparently better than that of patients with low RECK expression. Therefore, RECK is considered to be an cancer-inhibitory gene, which can affect tumor metastasis through inhibiting the activity of MMPs and angiogenesis.

\section{Innovations and breakthroughs}

No reports on this subject have been published in China or abroad. The immunohistochemistry SP method was used to perform a combined test on expression of RECK, VEGF and CD105 genes in 62 cases of ESCC, 31 specimens of para-carcinoma atypical hyperplastic tissues, and 62 specimens of normal esophageal mucous membrane, to ascertain the role of RECK, VEGF and CD105 in the generation and development of esophageal cancer, so as to establish a molecular index for early diagnosis and prognosis judgment.

\section{Applications}

The further investigation of RECK helps us understand more about the biological behavior of esophageal carcinoma and it gives us a new guide for earlier diagnosis and therapy of esophageal carcinoma. RECK may be a molecular target for the early diagnosis and prognostic judgment of esophageal carcinoma.

\section{Terminology}

RECK is a new cancer-inhibiting gene first discovered in NIH3T3 cell lines transfected by v-Ki-Ras.

\section{Peer review}

This manuscript reports expression of three genes in ESCC, in which they appear to have prognostic value as they are correlated with histological grade, lymphatic metastasis and invasion.

\section{REFERENCES}

1 Takahashi C, Sheng Z, Horan TP, Kitayama H, Maki M, Hitomi K, Kitaura Y, Takai S, Sasahara RM, Horimoto A, Ikawa Y, Ratzkin BJ, Arakawa T, Noda M. Regulation of matrix metalloproteinase-9 and inhibition of tumor invasion by the membrane-anchored glycoprotein RECK. Proc Natl Acad Sci USA 1998; 95: 13221-13226

2 Span PN, Sweep CG, Manders P, Beex LV, Leppert D, Lindberg RL. Matrix metalloproteinase inhibitor reversioninducing cysteine-rich protein with Kazal motifs: a prognostic marker for good clinical outcome in human breast carcinoma. Cancer 2003; 97: 2710-2715

3 Masui T, Doi R, Koshiba T, Fujimoto K, Tsuji S, Nakajima S, Koizumi M, Toyoda E, Tulachan S, Ito D, Kami K, Mori T, Wada M, Noda M, Imamura M. RECK expression in pancreatic cancer: its correlation with lower invasiveness and better prognosis. Clin Cancer Res 2003; 9: 1779-1784
4 da Silva Cardeal LB, Brohem CA, Corrêa TC, Winnischofer SM, Nakano F, Boccardo E, Villa LL, Sogayar MC, Maria-Engler SS. Higher expression and activity of metalloproteinases in human cervical carcinoma cell lines is associated with HPV presence. Biochem Cell Biol 2006; 84: 713-719

5 Chang HC, Cho CY, Hung WC. Downregulation of RECK by promoter methylation correlates with lymph node metastasis in non-small cell lung cancer. Cancer Sci 2007; 98: 169-173

6 Li SL, Zhao QM, Liu ZW, Zhao ZH, Gao DL, Zheng XY, Chen KS, Zhang YH .The correlation and clinical pathological significance of the expression of RECK and MMP-9 protein in esophageal squamous cell carcinoma. Shijie Huaren Xiaohua Zazhi 2007; 15: 1082-1086

7 Weidner N, Folkman J, Pozza F, Bevilacqua P, Allred EN, Moore DH, Meli S, Gasparini G. Tumor angiogenesis: a new significant and independent prognostic indicator in earlystage breast carcinoma. J Natl Cancer Inst 1992; 84: 1875-1887

8 Sasahara RM, Takahashi C, Noda M. Involvement of the Sp1 site in ras-mediated downregulation of the RECK metastasis suppressor gene. Biochem Biophys Res Commun 1999; 264 : 668-675

9 Chang HC, Cho CY, Hung WC. Silencing of the metastasis suppressor RECK by RAS oncogene is mediated by DNA methyltransferase $3 \mathrm{~b}$-induced promoter methylation. Cancer Res 2006; 66: 8413-8420

10 Takenaka K, Ishikawa S, Kawano Y, Yanagihara K, Miyahara R, Otake Y, Morioka Y, Takahashi C, Noda M, Wada H, Tanaka F. Expression of a novel matrix metalloproteinase regulator, RECK, and its clinical significance in resected nonsmall cell lung cancer. Eur J Cancer 2004; 40: 1617-1623

11 Noda M, Oh J, Takahashi R, Kondo S, Kitayama H, Takahashi C. RECK: a novel suppressor of malignancy linking oncogenic signaling to extracellular matrix remodeling. Cancer Metastasis Rev 2003; 22: 167-175

12 Sasahara RM, Brochado SM, Takahashi C, Oh J, Maria-Engler SS, Granjeiro JM, Noda M, Sogayar MC. Transcriptional control of the RECK metastasis/angiogenesis suppressor gene. Cancer Detect Prev 2002; 26: 435-443

13 Cho CY, Wang JH, Chang HC, Chang CK, Hung WC. Epigenetic inactivation of the metastasis suppressor RECK enhances invasion of human colon cancer cells. J Cell Physiol 2007; 213: 65-69

14 Liu LT, Chang HC, Chiang LC, Hung WC. Histone deacetylase inhibitor up-regulates RECK to inhibit MMP-2 activation and cancer cell invasion. Cancer Res 2003; 63: 3069-3072

15 Wang F, Wei L, Chen L. [The relationship between vascular endothelial growth factor, microvascular density, lymph node metastasis and prognosis of breast carcinoma]. Zhonghua Binglixue Zazhi 2000; 29: 172-175

16 Adams J, Carder PJ, Downey S, Forbes MA, MacLennan K, Allgar V, Kaufman S, Hallam S, Bicknell R, Walker JJ, Cairnduff F, Selby PJ, Perren TJ, Lansdown M, Banks RE. Vascular endothelial growth factor (VEGF) in breast cancer: comparison of plasma, serum, and tissue VEGF and microvessel density and effects of tamoxifen. Cancer Res 2000; 60: 2898-2905

17 Roncone D, Satoskar A, Nadasdy T, Monk JP, Rovin BH. Proteinuria in a patient receiving anti-VEGF therapy for metastatic renal cell carcinoma. Nat Clin Pract Nephrol 2007; 3: 287-293

18 Giralt J, Navalpotro B, Hermosilla E, de Torres I, Espin E, Reyes V, Cerezo L, de las Heras M, Ramon y Cajal S, Armengol M, Benavente S. Prognostic significance of vascular endothelial growth factor and cyclooxygenase- 2 in patients with rectal cancer treated with preoperative radiotherapy. Oncology 2006; 71: 312-319

19 Abe A, Fukui H, Fujii S, Fujita M, Mukawa K, Ichikawa K, Tomita S, Ono Y, Imai Y, Imura J, Kubota K, Fujimori T. Involvement of cyclooxygenase-2 and vascular endothelial growth factor in vascularization and lymph node metastasis of colorectal cancers with submucosal invasion. J Gastroenterol Hepatol 2007; 22: 1071-1077

20 Jang YJ, Kim DS, Jeon OH, Kim DS. Saxatilin suppresses 
tumor-induced angiogenesis by regulating VEGF expression in NCI-H460 human lung cancer cells. J Biochem Mol Biol 2007; 40: $439-443$

21 Nikiteas NI, Tzanakis N, Theodoropoulos G, Atsaves V, Christoni Z, Karakitsos P, Lazaris AC, Papachristodoulou A, Klonaris C, Gazouli M. Vascular endothelial growth factor and endoglin (CD-105) in gastric cancer. Gastric Cancer 2007; 10: 12-17

22 Hao L, Zhang C, Qiu Y, Wang L, Luo Y, Jin M, Zhang Y, Guo TB, Matsushima K, Zhang Y. Recombination of CXCR4, VEGF, and MMP-9 predicting lymph node metastasis in human breast cancer. Cancer Lett 2007; 253: 34-42

23 He SQ, Zhang WY. [Relationship between the expressions of KaI1, nm23, ETS-1, VEGF and microvascular density and clinical significance in nasopharyngeal carcinoma]. Zhonghua Erbi Yanhou Toujing Waike Zazhi 2006; 41: 813-817

24 El-Gohary YM, Silverman JF, Olson PR, Liu YL, Cohen JK, Miller R, Saad RS. Endoglin (CD105) and vascular endothelial growth factor as prognostic markers in prostatic adenocarcinoma. Am J Clin Pathol 2007; 127: 572-579

25 Gerber SA, Rybalko VY, Bigelow CE, Lugade AA, Foster TH, Frelinger JG, Lord EM. Preferential attachment of peritoneal tumor metastases to omental immune aggregates and possible role of a unique vascular microenvironment in metastatic survival and growth. Am J Pathol 2006; 169: 1739-1752

Chien CY, Su CY, Hwang CF, Chuang HC, Chen CM, Huang CC. High expressions of CD105 and VEGF in early oral cancer predict potential cervical metastasis. J Surg Oncol 2006; 94 413-417

27 Erdem O, Taskiran C, Onan MA, Erdem M, Guner H, Ataoglu O. CD105 expression is an independent predictor of survival in patients with endometrial cancer. Gynecol Oncol 2006; 103 1007-1011

28 Wikström P, Lissbrant IF, Stattin P, Egevad L, Bergh A Endoglin (CD105) is expressed on immature blood vessels and is a marker for survival in prostate cancer. Prostate 2002; $\mathbf{5 1}$ 268-275

29 Minhajat R, Mori D, Yamasaki F, Sugita Y, Satoh T, Tokunaga O. Organ-specific endoglin (CD105) expression in the angiogenesis of human cancers. Pathol Int 2006; 56: 717-723

30 Yang LY, Lu WQ, Huang GW, Wang W. Correlation between CD105 expression and postoperative recurrence and metastasis of hepatocellular carcinoma. BMC Cancer 2006; 6: 110

31 Xi YX, Song X, Chen HX, Peng TS, Chen MH. [Expression of microvessel density in multistep tissues of gastric carcinoma and significance thereof]. Zhonghua Yixue Zazhi 2006; 86: 3405-3408

32 Takeuchi T, Hisanaga M, Nagao M, Ikeda N, Fujii H, Koyama F, Mukogawa T, Matsumoto H, Kondo S, Takahashi C, Noda M, Nakajima Y. The membrane-anchored matrix metalloproteinase (MMP) regulator RECK in combination with MMP-9 serves as an informative prognostic indicator for colorectal cancer. Clin Cancer Res 2004; 10: 5572-5579

S- Editor Liu Y L- Editor Kerr C E- Editor Lu W 